\title{
LARGE DISKS AS REPRESENTATIONS FOR \\ THE LUNAR MASCONS WITH IMPLICATIONS REGARDING \\ THEORIES OF FORMATION
}

\author{
P. M. MULLER and W. L.SJOGREN \\ Jet Propulsion Laboratory, Pasadena, California, U.S.A.
}

\begin{abstract}
It is shown that large (hundred-km diam) near-surface disks are capable of accurately representing the lunar mascons with very few parameters. While this does not 'prove' that the mascons are excess mass in disk form, it is highly suggestive. It is pointed out that virtually every proposed mascon theory is consistent with a disk shape for the excess mass. The tentative hypothesis is advanced that the mascons are, in fact, disk shaped mass excesses. The conditions for mascon formation and preservation are reviewed in the light of this hypothesis.
\end{abstract}

\section{Introduction}

The resolution of Doppler tracking data from the Lunar Orbiter Mission to obtain lunar gravity information (Muller and Sjogren, 1968) was not sufficient to differentiate a point mass from a surface disk, as a model for a mascon. This was initially pointed out by Conel and Holstrom (1968). Kane (1969) showed that a low altitude orbit over various mascon models would indeed produce quite different gravity signatures. Apollos 12 and 14 have provided both high- and low- altitude orbits over the Nectaris mascon. Comparison of these gravity profiles with those of theoretical models is presented and some assertions are made.

\section{Approach}

The raw Doppler data from Apollos 12 and 14 were fit in short arcs (1 revolution or less) with the JPL orbit determination program. This program calculated the theoretical observations accounting for gravitation effects of the Sun, planets and a spherical Moon, the Earth's rotation, signal transit times, and atmospherics. It then did a leastsquares fit to the actual observations. The resulting residuals between the observation and theoretical calculations had characteristic signatures which were, in turn, differentiated to provide line-of-sight accelerations. These gravity profiles were plotted and represented the 'real world'.

The next step was to simulate tracking data for two different models. The first model was a point mass buried $100 \mathrm{~km}$ which represented possibly the remnants of an impactor. The second model was a surface disk which represented dense surface lava pools. The simulated data was then fit with the theoretical model used for the real Doppler observations (i.e., spherical Moon). The residuals were processed in the same manner as mentioned above and their gravity profiles were over-plotted with the initial 'real world' gravity profiles. Several attempts of modifying the model parameters to best match the real data residuals was done using Apollo 12 data. For 
example, the mass and depth of the point model were chosen to match the amplitude of the high- and low-altitude residuals. Likewise, the mass and radius of the surface disk were chosen so as to match these same amplitudes. Apollo 14 then was the independent test for these models. All the simulated data cases placed the perturbing mass at the center of Mare Nectaris ( -16 lat. and 34 long.). The point was $9.1 \times 10^{-6} G M_{\mathbb{}}$ at a depth of $100 \mathrm{~km}$, while the 150 and $200 \mathrm{~km}$ radius surface disks had masses of $5.1 \times 10^{-6} G M_{\mathbb{Q}}$ and $9.1 \times 10^{-6} G M_{\mathbb{Q}}$ respectively.

\section{Results}

The high-altitude gravity profiles are shown in Figure 1. The amplitudes of the point mass and $150 \mathrm{~km}$ disk match the real data fairly well, although the complete shape of the real data curve is somewhat broader. The $200 \mathrm{~km}$ disk seems much too large. The low-altitude profile in Figure 2 shows again the fairly good match of the real data amplitude with the point mass and the $150 \mathrm{~km}$ disk. However, the shape of both of them do not compare well at all. The point mass is much to narrow with its peak shifted $2^{\circ}$ east of the real peak. The $150 \mathrm{~km}$ disk is better, but still too narrow, with its peak $1 \frac{1}{2}^{\circ}$ east. The $200 \mathrm{~km}$ disk does a relatively good job in matching both amplitude and shape. A $200 \mathrm{~km}$ disk is physically pleasing, for it is just the radius of the prominent mare basin and the edge of Montes Pyrenaeus.

The Apollo 14 low altitude data provides another comparison of models as shown in Figure 3. Again the point mass is the poorest fit; the $150 \mathrm{~km}$ disk is somewhat better, but is still too narrow; and the $200 \mathrm{~km}$ disk has about the right shape but is slightly over in amplitude.

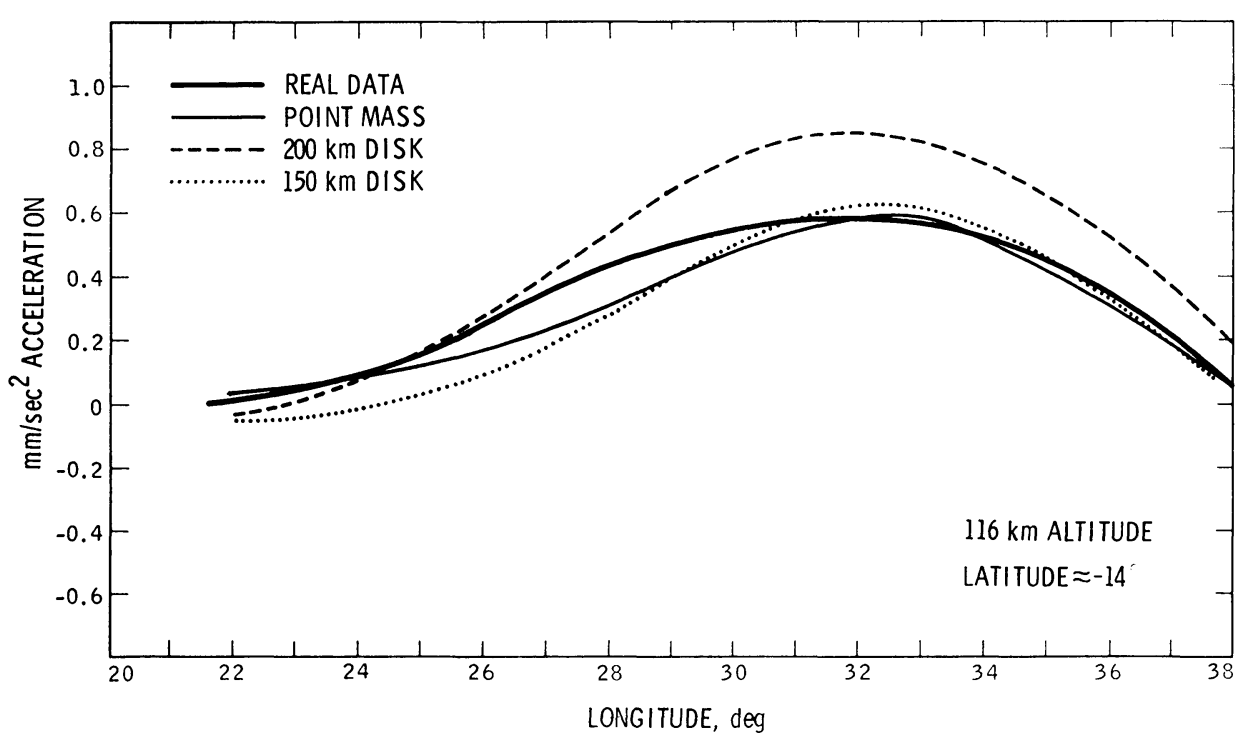

Fig. 1. Nectaris gravity profile by Apollo 12 at $116 \mathrm{~km}$ altitude. 


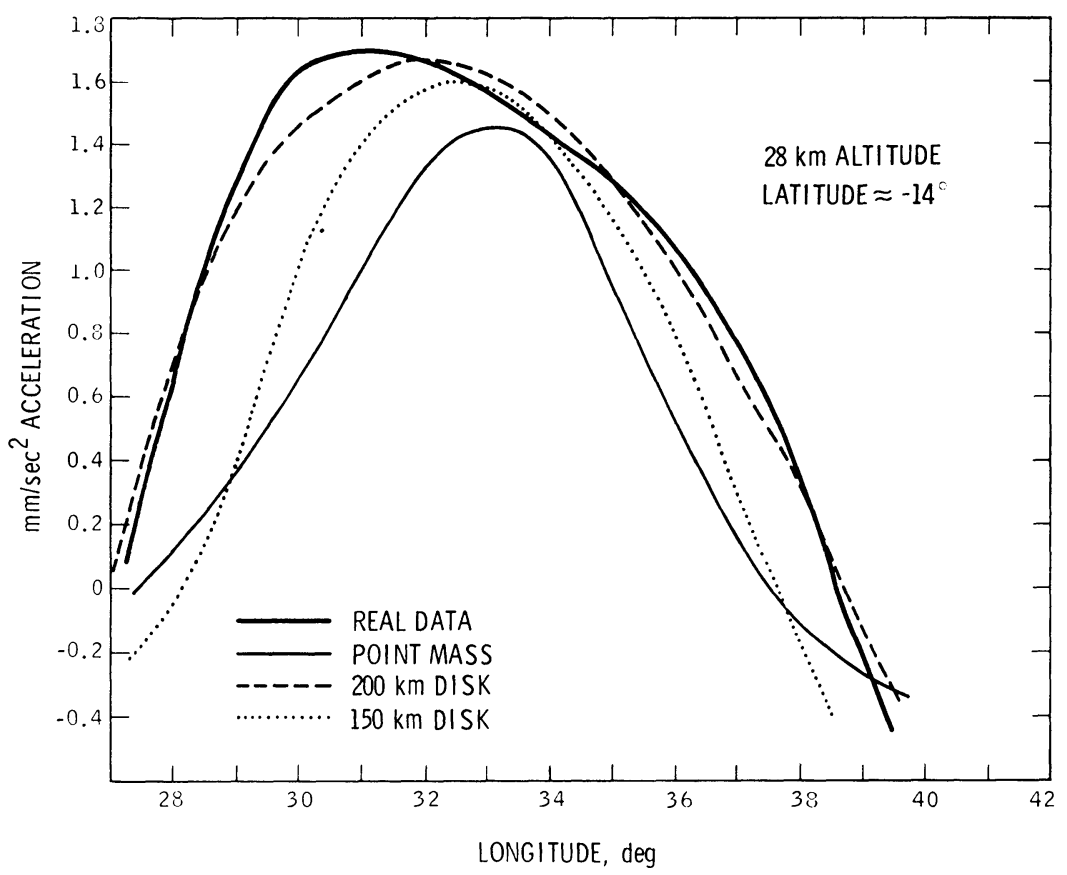

Fig. 2. Nectaris gravity profile by Apollo 12 at $28 \mathrm{~km}$ altitude.

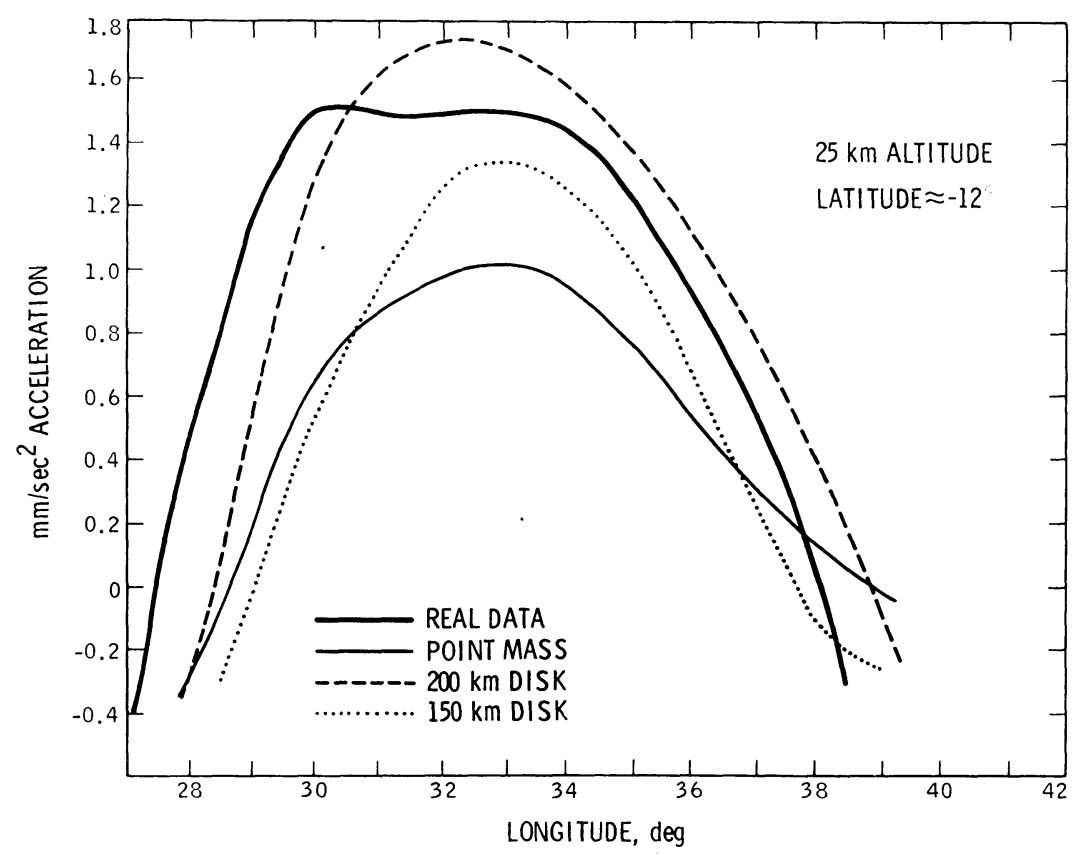

Fig. 3. Nectaris gravity profile by Apollo 14 at $25 \mathrm{~km}$ altitude. 


\section{Conclusion}

From the comparisons in Figures 1-3, it is evident that disks do a much better job of representing the real data than does a buried point mass. However, it is not clear that a simple disk can completely model the real data. This is the case with the $200 \mathrm{~km}$ disk which does well in low altitude orbits but rather poorly for high altitude data. There may be some compensating negative gravity anomalies adjacent to the mascon area that averages in high altitude data and thus reduces the positive gravity as presented in the theoretical model. A more quantitative answer will be forthcoming with the reduction of all the Apollo 14 data, from which the masses for a dense grid of surface disks will be estimated over a broad area (i.e., not just the Nectaris central basin) in a dynamical least squares fit.

In theory, no amount of above-surface gravity measurements can define a subsurface mass distribution, as there are an infinite number of distributions which can fulfill the observations. In practice, as any geologist can testify, many successful deductions are in fact made from above-surface gravimetry. Few oil companies could make a profit from wildcat drilling were it not for this successful generalization from insufficient observations. It has been shown above that the spherical mass point model does not fit the observations nearly so well as the disk, despite the fact that the disk has 'sharp' edges which the spacecraft overflies. In the hand-working of even some lunar orbiter data, we have noted many cases of 'shoulders' near the edges of mascon seas, and have speculated on disk models. It appears safe to at least advance the hypothesis that the mascons are, in fact, disk shaped mass excesses, and to take note of the indirect but highly suggestive consistency of observations with mass-models of this shape and nature.

\section{Theories of Mascon Formation and Mascon Shape}

A brief summary of current mascon formation theories is given below, and it is noted that all of them produce mass excess in approximate disk shapes near the lunar surface. The hypothesis that the mascons are, in fact, disk shaped, obtains indirect support from the fact that all physical theories of mascon formation advanced to date do require or produce this shape. Conversely, the observed gravity data have been shown to be consistent with the disk shape.

Fundamental physical analysis results in three physical requirements for formation and preservation of a lunar mascon, which we note is characterized by higher gravity over a topographic low. The presence of a mascon implies: (1) the presence of higher density material in the basin; (2) there is added mass in the basin being supported above the point of isostatic equilibrium; (3) this support of mass above isostatic equilibrium has been maintained (presumably) for geologic time. (Table I.)

The authors have synthesized a five-step mascon formation schematic, within which the major mascon formation theories can be analyzed (Gottlieb and Muller, in press). Table II presents this schematic theory of mascon formation. Steps 1 and 2 are 


\section{TABLE I}

Immediate mascon conclusions

Observation: Higher gravity over topographic lows (basins).

Requirement: Presence of higher density material.

Requirement: Presence of mass supported above isostasy.

Requirement: That the mass was supported for geologic time (stress 50-400 bars).

Conclusion: Some mechanism produced very large volumes of significantly higher density material in the ringed sea basins.

Conclusion: Some process raised very large volumes of lunar mass above the level of isostatic equilibrium (approximately $3 \mathrm{~km}$ above this level).

Conclusion: The Moon retained sufficient strength to a depth of at least $100 \mathrm{~km}$ to support a stress of 50 to 400 bars for geologic time.

Conclusion: The temperature of the lunar material to this depth could hardly have risen to $800^{\circ} \mathrm{C}$ and if this high, could only have remained hot for a geologically short time $\left(10^{3}\right.$ to $\left.10^{6} \mathrm{yrs}\right)$.

generally agreed to have taken place - particularly, step 2, which was rather firmly established by Harold Urey after W. C. Gilbert. If step 3 did not take place, then very high density material had to be transported in large quantities. H. C. Urey's suggestion (Table III) of direct formation by impact combines steps 3 and 2, and avoids any problem with steps 4 and 5 . It is the simplest explanation, but suffers from apparent theoretical difficulties in accounting for the fact that the asteroidal body is retained after the tremendous release of impact energy. If his hypothesis is simply accepted, however, the resulting mascon will be disk shaped, because a buried sphere is physically impossible, and the shattered asteroidal remains would surely be scattered in the basin bottom. Both the lava-flow and transport models are basically similar, except the latter avoids temperature problems that raise strong doubts in some minds as to

TABLE II

Schematic theory of mascon formation.

Step 1 The Moon forms a crust of lower density than the interior. The thickness must be at least $25 \mathrm{~km}, 50 \mathrm{~km}$ most likely, $100 \mathrm{~km}$ likely maximum. This constraint is placed by the next step.

Step 2 An asteroidal-sized body impacts at the future location of a mascon sea, blasting crustal material away to a depth sufficient for the next step.

Step 3 The initial deep crater is back-filled from below with the higher-density material until approximate isostasy is achieved. A shallow basin of 5-10 km depth remains due to the density contrast of the crust versus the interior.

Step 4 The material below the basin floor now achieves and retains sufficient strength to withstand a shear force of 50-400 bars for geologic time.

Step 5 The basin is further filled with material sufficient to give the observed positive gravity anomaly (above isostasy). Any slumping of the floor removes the anomaly, either during or following the deposition. The effective density contrast for this step is approximately 3.0 , since vacuum is being replaced with rock. 
TABLE III

Basic classes of mascon theories

Lavas: Wise, Yates, and Booker, Kovach, Lu and Rell: Fall into two classes:

Lavas perfuse upward through a crust able to withstand the shear.

Lavas are deposited in the basin by flows from outside the basin.

Physical dynamics of a shrinking Moon: Kaula:

The lunar crust shrinks, does not crack, and forces lavas above isostasy into the basins.

Transport of material into compensated basin: Gilvarry, Gold, others:

Transport of sediments by water in a lunar hydrosphere.

Transport of lunar fines or dust by various mechanisms.

Transport by any other scheme.

Direct formation by impact: Urey

The asteroidal impacting body which formed the seas is of higher density than the Moon and leaves behind more mass than was ejected.

Other theories so far offered fail to explain the super-isostatic condition.

whether the sub-basin materials could simultaneously transmit large quantities of hot lavas and still support the mascon stress of 50-400 bars for even very short geologic time spans.

Several theories of mascon formation have been proposed which fail to account for the super-isostatic condition of the excess mass. It can easily be shown (Gottlieb and Muller, in press) that isostatic mascon models are impossible or impractical for reasonable density contrasts. The three requirements given in Table I are rather firmly advanced as requirements on any theory of mascon formation. When we examine each theory noted in Table III, it is evident that each produces a roughly disk-shaped excess of mass near the surface.

It is, therefore, the strong suggestion of this paper that the mascons are, in fact, near-surface, disk-shaped mass distributions. Since there are no reasonable experiments that can be conducted to firmly establish this hypothesis, and since this model is consistent with the observations and all theories advanced to date, we would appear safe in adopting the hypothesis. Further, we can add a fourth 'requirement' on any future mascon theory; namely, that it either be consistent with the near-surface disk distribution profile, or be carefully tested against the far higher resolution, low-altitude data being currently obtained from Apollo tracking, which does seem capable of discriminating at least between widely different mass distribution models such as point mass at depth, surface disks, and perhaps others.

\section{References}

Conel, J. E. and Holstrom, G. B.: 1968, Science 162, 1403.

Gottlieb, P. and Muller, P. M.: in Proc. of the Lunar Science Institute Symposium on the Geophysical Interpretation of the Moon, Houston, Texas, June 1970 (to be published).

Kane, M.: 1969, J. Geophys. Res. 74, 6579.

Muller, P. M. and Sjogren, W.L.: 1968, Science 161, 680. 